\title{
INTERVALO HÍDRICO ÓTIMO EM UM LATOSSOLO VERMELHO DISTROFÉRRICO SOB DIFERENTES SISTEMAS DE PRODUÇÃOO ${ }^{1}$
}

\author{
MILSON E. SERAFIM ${ }^{2}$, ANTÔNIO C. T. VITORINO ${ }^{3}$, PAULA P. P. PEIXOTO ${ }^{3}$, \\ CRISTIANO M. A. SOUZA ${ }^{3}$, DANIEL F. DE CARVALHO ${ }^{4}$
}

\begin{abstract}
RESUMO: Este trabalho teve o objetivo de caracterizar sistemas de cultivo quanto à qualidade estrutural do solo para o desenvolvimento de plantas por meio da determinação do Intervalo Hídrico Ótimo (IHO). O estudo foi realizado com amostras indeformadas de um Latossolo Vermelho distroférrico, sob os sistemas de cultivo convencional, direto e integração lavourapecuária, no município de Dourados - MS. Em cada sistema de cultivo, foram amostrados 28 pontos, nas camadas de 0-0,05 m, 0,05-0,10 m, e 0,10-0,20 m, totalizando 84 amostras por sistema, que foram utilizadas para a determinação da curva de retenção de água no solo, da curva de resistência do solo à penetração e da porosidade. No sistema de cultivo convencional e no direto, a menor limitação ao desenvolvimento radicular foi na camada de $0-0,05 \mathrm{~m}$, e na integração, a limitação foi menor na camada de $0,10-0,20 \mathrm{~m}$. Na camada de $0-0,05 \mathrm{~m}$ do convencional, o IHO foi igual à capacidade de água disponível. Nos sistemas direto e integração, a resistência à penetração foi limitante. A resistência à penetração foi o principal fator limitante do IHO na maioria das situações estudadas.
\end{abstract}

PALAVRAS-CHAVE: integração lavoura-pecuária, plantio direto, água no solo.

\section{LEAST LIMITING WATER RANGE IN A DISTROFERRIC RED LATOSSOL UNDER DIFFERENT GROWING SYSTEMS}

\begin{abstract}
The objective of this work was to characterize the growing systems related to the structural soil quality for plants development by the Least Limiting Water Range (LLWR). The study was developed using soil samples with preserved structure of a Distroferric Red Latossol under the conventional, no tillage and integrated growing systems in de county of Dourados-MS, Brazil. In each system 28 points were sampled, in 0-0.05, 0.05-0.10 and 0.10-0.20 m deep layers, ending up with 84 samples by system which was used to adjust the water retention curve in the soil, soil resistance to penetration curve and porosity determination, all of them necessary to the LLWR determination. In the conventional and no-tillage systems the smallest restriction to de roots development was in the $0-0.05 \mathrm{~m}$ layer, while in the integration system the smallest limiting was in $0.10-0.20 \mathrm{~m}$ layer. In the conventional system, in the $0-0.05 \mathrm{~m}$ layer, the LLWR was equal to the available water capacity, in the direct and integrated systems the resistance to penetration was the limiting factor. The resistance to penetration was the major limiting factor in most of the studied situations.
\end{abstract}

KEYWORDS: integration, no-tillage system, water in soil.

\footnotetext{
${ }^{1}$ Extraído da Dissertação de Mestrado do primeiro autor.

${ }^{2}$ Mestre em Agronomia, Doutorando em Ciência do Solo, UFLA, Lavras - MG, milsonserafim@gmail.com

${ }^{3}$ Faculdade de Ciências Agrárias, UFGD, Dourados - MS

${ }^{4}$ Instituto de Tecnologia, Departamento de Engenharia, UFRRJ, Seropédica - RJ, carvalho@ ufrrj.br. Bolsista do CNPq.

Recebido pelo Conselho Editorial em:16-10-2007

Aprovado pelo Conselho Editorial em: 10-10-2008
} 


\section{INTRODUÇÃO}

No escopo da física do solo, um dos problemas relevantes é a compactação do solo, especialmente com a grande expansão de sistemas de cultivos com revolvimento mínimo do solo, representado, genericamente, pelo sistema plantio direto (SPD) em substituição ao sistema convencional de preparo do solo.

Latossolos com mineralogia oxídica predominante na fração argila tendem a apresentar menor capacidade de armazenamento de água (FERREIRA et al., 1999) e em condições de ocorrência de veranicos, estiagens, grande demanda evapotranspirométrica, e a distribuição irregular das chuvas pode resultar na ocorrência de déficit hídrico, que é uma das principais causas de perdas agrícolas na safra de verão na região de Dourados - MS (FIETZ \& URCHEI, 2002). Esses fatores destacam a importância do solo como reservatório de água para as plantas, o qual deve possuir condições de armazenamento hídrico dentro da faixa de tensão utilizada pela cultura, cujo limite superior é dado pela capacidade de campo $(0,01 \mathrm{MPa})$ e o limite inferior pelo ponto de murcha permanente (1,5 MPa) (CAVALIERI et al., 2006).

O conceito de "Least Limiting Water Range" (LLWR) foi proposto por LETEY (1985) e desenvolvido por SILVA \& KAY (1994) como indicador da qualidade estrutural do solo para o crescimento das raízes, visto que integra, num único parâmetro, os três fatores importantes associados ao desenvolvimento das plantas: aeração, umidade e resistência mecânica do solo. Pesquisas têm mostrado que o LLWR, traduzido como Intervalo Hídrico Ótimo (IHO) (TORMENA et al., 1998), é alterado pelo grau de compactação do solo (SILVA \& KAY, 1997; BETZ et al., 1998; IMHOFF et al., 2001; TORMENA et al., 2007).

Considerando a importância dos atributos físicos na dinâmica da água no solo e no desenvolvimento radicular das culturas, desenvolveu-se este trabalho com o objetivo de estudar o Intervalo Hídrico Ótimo em Latossolo Vermelho distroférrico, sob os sistemas de cultivo convencional, plantio direto e integração lavoura-pecuária.

\section{MATERIAL E MÉTODOS}

O trabalho foi realizado utilizando-se de amostras de um Latossolo Vermelho distroférrico muito argiloso (LVDf), com a seguinte composição granulométrica: areia $=280 \mathrm{~g} \mathrm{~kg}^{-1}$, silte $=$ $110 \mathrm{~g} \mathrm{~kg}^{-1}$ e argila $=610 \mathrm{~g} \mathrm{~kg}^{-1}$; localizado na Embrapa Agropecuária Oeste (CPAO), no município de Dourados - MS, no ano agrícola de 2005/2006. Nessa área, vem sendo conduzido experimento de longa duração (8 anos) com três sistemas de cultivo: 1) sistema convencional (SC); 2) sistema plantio direto (SPD); 3) sistema integração lavoura-pecuária (SL/P). As análises laboratoriais foram realizadas no Laboratório de Física do Solo, da Faculdade de Ciências Agrárias, da Universidade Federal da Grande Dourados.

Em cada um dos três sistemas, foram coletadas amostras indeformadas com anéis volumétricos $\left(100 \mathrm{~cm}^{3}\right)$ em 28 pontos aleatórios. Em cada ponto, foram amostradas três profundidades (0-5; 5-10 e 10-20 cm), perfazendo o total de 84 amostras por sistema de cultivo. Para determinar a curva de retenção de água (CRA), foi adotado o procedimento descrito em SILVA \& KAY (1994). As 28 amostras foram divididas em sete grupos de quatro amostras, sendo cada grupo submetido aos seguintes potenciais na câmara de Richards: $-0,004$; $-0,01$; $-0,033 ;-0,066 ;-0,1 ;-0,3$ e -1,5 MPa, conforme KLUTE (1986).

Quando as amostras atingiram o equilíbrio, foi medida a resistência do solo à penetração, utilizando penetrógrafo eletrônico, com velocidade constante de penetração de $10 \mathrm{~mm} \mathrm{~min}{ }^{-1}$, diâmetro de base da haste de $4 \mathrm{~mm}$ e semiângulo de $30^{\circ}$. A frequência de leitura de resistência à penetração correspondeu à coleta de um valor a cada 0,25 segundos, obtendo-se o total de 800 leituras por amostra, das quais se utilizou um valor médio. Determinada a resistência do solo à penetração, as amostras foram secas em estufa a $105-110{ }^{\circ} \mathrm{C}$, por $48 \mathrm{~h}$, para a determinação da densidade do solo pelo método do anel volumétrico (EMBRAPA, 1997). 
A porosidade de aeração, ou conteúdo de água que corresponde a uma porosidade livre para aeração de $10 \%$, foi estimada empregando a eq.(1):

$$
\mathrm{P}_{\mathrm{a}}=\mathrm{P}_{\mathrm{t}}-0,1
$$

em que,

$\mathrm{P}_{\mathrm{a}}$ - porosidade de aeração, $\mathrm{cm}^{3} \mathrm{~cm}^{-3}, \mathrm{e}$

$\mathrm{P}_{\mathrm{t}}$ - porosidade total, $\mathrm{cm}^{3} \mathrm{~cm}^{-3}$.

Para obter a curva de retenção de água no solo, foi utilizado o modelo linearizado (eq.(2)), adaptado de SILVA \& KAY (1994):

$$
\ln \theta=\mathrm{a}_{0}+\mathrm{a}_{1} \ln \mathrm{Ds}+\left(\mathrm{b}_{0}+\mathrm{b}_{1} \ln \mathrm{Ds}\right) \ln \mathrm{T}
$$

em que,

$\theta$ - umidade volumétrica, $\mathrm{cm}^{3} \mathrm{~cm}^{-3}$;

Ds - densidade do solo, $\mathrm{Mg} \mathrm{m}^{-3}$;

$\mathrm{T}$ - potencial mátrico, MPa, e

$a_{0}, a_{1}, b_{0}$ e $b_{1}$ - coeficientes obtidos com ajuste dos modelos.

Para a estimativa da capacidade de campo (CC) e do ponto de murcha permanente (PMP), utilizou-se da eq.(2), considerando a tensão em -0,01 e -1,5 MPa para CC e PMP, respectivamente.

A curva de resistência do solo à penetração foi ajustada por meio de modelo não-linear, proposto por BUSSCHER (1990):

$$
\mathrm{RP}=\mathrm{a}+\theta^{\mathrm{b}}+\mathrm{Ds}^{\mathrm{c}}
$$

em que,

$\mathrm{RP}$ - resistência do solo à penetração, $\mathrm{MPa}$;

$\theta$ - umidade volumétrica, $\mathrm{m}^{3} \mathrm{~m}^{-3}$;

Ds - densidade do solo, $\mathrm{Mg} \mathrm{m}^{-3}$, e

a, b e c - coeficientes obtidos por meio do ajuste dos modelos.

O IHO foi determinado com base nos procedimentos descritos em SILVA et al. (1994). Os valores críticos de umidade associados com o potencial mátrico, resistência do solo à penetração e porosidade de aeração foram, respectivamente: capacidade de campo $\left(\theta_{\mathrm{cc}}\right)$, potencial de $-0,01 \mathrm{MPa}$ (CAVALIERI et al., 2006); ponto de murcha permanente $\left(\theta_{\mathrm{PMP}}\right)$, potencial de $-1,5 \mathrm{MPa}$ (CAVALIERI et al., 2006); umidade volumétrica, em que a resistência do solo à penetração $\left(\theta_{\mathrm{RP}}\right)$ atinge 2,0 MPa (TAYLOR et al., 1966), e a umidade volumétrica na porosidade de aeração ( $\left.\theta_{\mathrm{PA}}\right)$, $0,10 \mathrm{~m}^{3} \mathrm{~m}^{-3}$ (GRABLE \& SIEMER, 1968).

A Figura do IHO, em cada sistema de cultivo, foi obtida plotando-se a eq.(2) com valor de T de -10 e $-1.500 \mathrm{kPa}$, para a capacidade de campo e ponto de murcha permanente, respectivamente, mais as eqs. (1) e (3) em um mesmo sistema de coordenadas x e y. No eixo das abscissas, estão os valores crescentes de densidade do solo entre o mínimo e o máximo valor de densidade encontrado no sistema de cultivo. O eixo y representa a umidade do solo.

Os modelos de retenção de água no solo e da resistência à penetração foram selecionados com base no erro relativo médio e no teste estatístico F, e seus coeficientes obtidos utilizando-se do método de Gauss-Newton, usado para a minimização do quadrado dos resíduos. Na comparação entre os dados estimados e os observados, adotou-se o método estatístico proposto por LEITE \& OLIVEIRA (2002), utilizando-se do teste F modificado por Graybill (eq.(4)) para analisar a correlação linear entre os dados estimados e os observados, e o teste $t$ aplicado ao erro relativo médio (eq.(5)). 


$$
\begin{aligned}
& \mathrm{F}\left(\mathrm{H}_{0}\right)=\frac{(\beta-0)^{\prime}\left(\mathrm{Y}_{1}^{\prime} \mathrm{Y}_{1}\right)(\beta-\theta)}{2 \mathrm{QMR}} \sim \mathrm{F}_{\alpha}(2, \mathrm{n}-2) \\
& t_{\overline{\mathrm{e}}}=\frac{\overline{\mathrm{e}}-0}{\mathrm{~S}_{\overline{\mathrm{e}}}} \sim \mathrm{t}_{\alpha}(\mathrm{n}-1)
\end{aligned}
$$

sendo:

$$
\mathrm{S}_{\overline{\mathrm{e}}}=\frac{\mathrm{S}_{\mathrm{e}}}{\sqrt{\mathrm{n}}}
$$

em que,

$\mathrm{Y}_{1}$ - vetor de valores observados;

$\beta$ - vetor de coeficientes $\left(\beta_{0}\right.$ e $\left.\beta_{1}\right)$;

QMR - quadrado médio do resíduo da regressão;

$\overline{\mathrm{e}}$ - erro relativo médio;

$\mathrm{S}_{\overline{\mathrm{e}}}$ - desvio-padrão do erro médio;

$S_{\mathrm{e}}$ - desvio-padrão;

$\mathrm{n}$ - número de observações;

$\alpha$ - significância do teste $\mathrm{F}$;

$\mathrm{t}_{\overline{\mathrm{e}}}-$ valor do teste $\mathrm{t}$ do erro médio, $\mathrm{e}$

$\mathrm{H}_{0}-\beta^{\prime}=\left[\begin{array}{ll}0 & 1\end{array}\right]$

O erro relativo médio entre os dados estimados e os observados foi determinado utilizando-se da eq.(7). Considerando-se que o erro (eq.(8)) é uma variável que segue distribuição normal e ocorre ao acaso, pode-se testar a hipótese $\left(\mathrm{H}_{0}: \overline{\mathrm{e}}=0\right)$, que o erro relativo médio é zero:

$$
\begin{aligned}
& \overline{\mathrm{e}}=\frac{\sum_{\mathrm{i}}^{\mathrm{n}} \frac{\mathrm{Y}_{\mathrm{ji}}-\mathrm{Y}_{1 \mathrm{i}}}{\mathrm{Y}_{1 \mathrm{i}}}}{\mathrm{n}} \\
& \mathrm{e}=\frac{\mathrm{Y}_{\mathrm{j}}-\mathrm{Y}_{1}}{\mathrm{Y}_{1}}
\end{aligned}
$$

em que,

$\mathrm{Y}_{\mathrm{j}}$ - vetor de valores estimados;

e - erro, e

$\mathrm{i}-1,2, \ldots, \mathrm{n}$.

Foram utilizados $5 \%$ de probabilidade nas comparações entre os dados obtidos experimentalmente e aqueles estimados dos modelos.

\section{RESULTADOS E DISCUSSÃO}

Os atributos físicos empregados no ajuste dos modelos de retenção de água e de resistência do solo à penetração são apresentados na Tabela 1. A densidade do solo e a retenção de água no solo estão de acordo com LEÃO (2002), que trabalhou com Latossolo Vermelho distróférrico. É possível verificar que o coeficiente de variação da resistência à penetração foi o mais elevado entre os atributos avaliados em virtude de sua grande variabilidade. TORMENA et al. (1998) comentam que esse comportamento pode estar relacionado à variabilidade natural da densidade do solo, ao gradiente de umidade entre as amostras e aos diferentes sistemas de cultivo que foram amostrados. 
TABELA 1. Estimativa da umidade volumétrica $\left(\theta, \mathrm{em} \mathrm{m}^{3} \mathrm{~m}^{-3}\right.$ ), da densidade (Ds, em $\mathrm{Mg} \mathrm{m}^{-3}$ ) e da resistência à penetração ( $\mathrm{RP}$, em $\mathrm{MPa}$ ), determinadas nas amostras de solo com estrutura indeformada. Estimation of water content $\left(\theta \mathrm{m}^{3} \mathrm{~m}^{-3}\right)$, the density (Ds, in $\mathrm{Mg} \mathrm{m}^{-3}$ ) and of resistance to penetration (PR, in MPa), certain soil samples with preserved structure.

\begin{tabular}{lccccc}
\hline Atributo & Média & Desvio-padrão & C.V. & Mínimo & Máximo \\
\hline$\theta$ & 0,353 & 0,063 & 18 & 0,219 & 0,497 \\
Ds & 1,126 & 0,128 & 11 & 0,772 & 1,363 \\
RP & 2,059 & 1,482 & 72 & 0,100 & 6,921 \\
\hline
\end{tabular}

$\overline{\text { C.V. }}=$ coeficiente de variação $(\%)$.

Os coeficientes do modelo de retenção de água no solo são apresentados na Tabela 2. Pode-se observar que os modelos apresentaram elevados coeficientes de determinação e não foi observada significância do teste $\mathrm{t}$ do erro relativo médio e do teste $\mathrm{F}$ dos coeficientes de correlação, garantindo identidade dos modelos estimados com o comportamento dos dados experimentais.

TABELA 2. Valores dos coeficientes dos modelos de retenção de água no solo $\left(a_{0}, a_{1}, b_{0}\right.$ e $\left.b_{1}\right)$, dos coeficientes de determinação $\left(R^{2}\right)$, do teste $t$ dos erros relativos médios $\left(t_{\bar{e}}\right)$ e do teste $\mathrm{F}$, em cada profundidade (m) e sistema de cultivo. Values of the coefficients of the models of water retention in the soil $(\mathrm{a} 0, \mathrm{a} 1, \mathrm{b0}$ and $\mathrm{B} 1)$, the coefficients of determination $\left(R^{2}\right)$, the test $t$ of errors concerning average $\left(t_{\bar{e}}\right)$ and the $F$ test, in each depth (m) and cultivation system.

\begin{tabular}{cccrccccc}
\hline Camada (m) & Sistemas & $\mathrm{a}_{0}$ & \multicolumn{1}{c}{$\mathrm{a}_{1}$} & $\mathrm{~b}_{0}$ & $\mathrm{~b}_{1}$ & $\mathrm{R}^{2}$ & $\mathrm{t}_{\overline{\mathrm{e}}}{ }^{*}$ & $\mathrm{~F}_{\left(\mathrm{H}_{0}\right)^{*}}$ \\
\hline \multirow{3}{*}{$0-0,05$} & Convencional & $-0,92220$ & 0,26620 & $-0,05907$ & 0,11434 & 0,83 & 0,55 & 1,30 \\
& Plantio Direto & $-0,71701$ & $-0,07584$ & $-0,11991$ & 0,25105 & 0,97 & 0,04 & 0,45 \\
& Integração & $-0,49918$ & $-1,83577$ & $-0,17085$ & 0,56816 & 0,70 & 0,69 & 0,44 \\
\hline \multirow{2}{*}{$0,05-0,10$} & Convencional & $-0,74489$ & 0,37088 & $-0,09372$ & 0,00288 & 0,94 & 1,46 & 0,94 \\
& Plantio Direto & $-1,06550$ & 2,65961 & 0,00959 & $-0,61711$ & 0,99 & 2,28 & 3,63 \\
& Integração & $-0,90980$ & 1,05586 & $-0,00764$ & $-0,40203$ & 0,90 & 1,53 & 0,97 \\
\hline \multirow{2}{*}{$0,10-0,20$} & Convencional & $-0,40500$ & $-1,34569$ & $-0,17372$ & 0,46009 & 0,94 & 1,12 & 0,65 \\
& Plantio Direto & $-0,86201$ & 0,86716 & $-0,06677$ & $-0,09831$ & 0,90 & 0,16 & 0,35 \\
& Integração & $-1,10926$ & 3,61685 & 0,03029 & $-0,96353$ & 0,83 & 0,34 & 1,26 \\
\hline
\end{tabular}

* Sem significância $(\mathrm{P}>5 \%)$

Os coeficientes dos modelos da resistência do solo à penetração são apresentados na Tabela 3. O modelo adaptado de SILVA \& KAY (1994), empregado no ajuste dos modelos de retenção de água no solo, estimou satisfatoriamente os valores de umidade, considerando significância de 5\% de probabilidade e o coeficiente de determinação. Verificou-se que a resistência do solo à penetração apresentou variação diretamente proporcional com a densidade do solo e inversamente proporcional com a umidade. Resultados semelhantes foram obtidos por ARAÚJO et al. (2005), LEÃO (2002) e TORMENA et al. (1998), para a mesma classe de solo.

As estimativas da umidade volumétrica em que a resistência à penetração atinge $2 \mathrm{MPa}$, empregando os coeficientes apresentados na Tabela 3, foram obtidas pela eq. (3), fixando o valor da resistência do solo à penetração em $2 \mathrm{MPa}$. 
TABELA 3. Valores dos coeficientes do modelo de resistência do solo à penetração (a, b e c), do coeficiente de determinação $\left(R^{2}\right)$, do teste $t$ do erro relativo médio e do teste $F$, em cada profundidade $(\mathrm{m})$ e sistema de cultivo. Values of the coefficients of the model of resistance of the soil to penetration $(a, b$ and $c)$, the coefficient of determination $\left(R^{2}\right)$, the $t$ test the relative medium error and the $F$ test, in each depth $(m)$ and cultivation system.

\begin{tabular}{cccccccc}
\hline Camada $(\mathrm{m})$ & Sistemas & $\mathrm{a}$ & $\mathrm{b}$ & $\mathrm{c}$ & $\mathrm{R}^{2}$ & $\mathrm{t}_{\overline{\mathrm{e}}}{ }^{*}$ & $\mathrm{~F}\left(\mathrm{H}_{0}\right)^{*}$ \\
\hline \multirow{2}{*}{$0-0,05$} & Convencional & 0,00805 & $-3,32149$ & 0,47717 & 0,84 & $1,06^{\mathrm{ns}}$ & $1,42^{\mathrm{ns}}$ \\
& Plantio direto & 0,00560 & $-4,26470$ & 1,76095 & 0,86 & $1,28^{\mathrm{ns}}$ & $0,70^{\mathrm{ns}}$ \\
& Integração & 0,00386 & $-3,90060$ & 12,78301 & 0,87 & $0,63^{\mathrm{ns}}$ & $0,16^{\mathrm{ns}}$ \\
\hline \multirow{3}{*}{$0,05-0,10$} & Convencional & 0,15404 & $-2,14546$ & 0,44684 & 0,64 & $1,03^{\mathrm{ns}}$ & $2,03^{\mathrm{ns}}$ \\
& Plantio Direto & 0,04812 & $-2,23779$ & 11,88776 & 0,85 & $0,86^{\mathrm{ns}}$ & $0,76^{\mathrm{ns}}$ \\
& Integração & 0,02409 & $-2,40166$ & 13,91457 & 0,89 & $1,30^{\mathrm{ns}}$ & $3,48^{\mathrm{ns}}$ \\
\hline \multirow{3}{*}{$0,10-0,20$} & Convencional & 0,08403 & $-2,31811$ & 5,09754 & 0,71 & $1,65^{\mathrm{ns}}$ & $0,59^{\mathrm{ns}}$ \\
& Plantio direto & 0,15125 & $-2,49688$ & 0,55096 & 0,75 & $1,26^{\mathrm{ns}}$ & $1,27^{\mathrm{ns}}$ \\
& Integração & 0,07786 & $-2,09104$ & 9,44577 & 0,57 & $1,25^{\mathrm{ns}}$ & $2,69^{\mathrm{ns}}$ \\
\hline
\end{tabular}

* Sem significância $(\mathrm{P}>5 \%)$.

Os valores de porosidade total e densidade do solo são apresentados na Tabela 4. A porosidade total foi máxima na camada de $0-0,05 \mathrm{~m}$, no sistema convencional, e teve seu menor valor na camada de 0,05-0,10 m, no sistema plantio direto, estando de acordo com CONTE et al. (2007). Para a densidade, o valor mínimo foi na camada de 0-0,05 m do convencional, devido ao efeito do revolvimento, e o valor máximo na camada de 0,10-0,20 $\mathrm{m}$ do plantio direto, provavelmente pelo efeito do tráfego de máquinas e menor matéria orgânica.

TABELA 4. Porosidade total $\left(\mathrm{P}_{\mathrm{t}}\right)$ e densidade do solo nas respectivas camadas $(\mathrm{m})$ e sistemas de cultivo, em um Latossolo Vermelho distroférrico. Total porosity $\left(\mathbf{P}_{\mathbf{t}}\right)$ and density of soil in their layers (m) and cultivation systems, in an Distroferric Red Latossol.

\begin{tabular}{cccc}
\hline Camada $(\mathrm{m})$ & Sistema & $\mathrm{P}_{\mathrm{t}}\left(\mathrm{cm}^{3} \mathrm{~cm}^{-3}\right)$ & $\mathrm{Ds}\left(\mathrm{cm}^{3} \mathrm{~cm}^{-3}\right)$ \\
\hline \multirow{3}{*}{$0-0,05$} & Convencional & 0,65 & 0,94 \\
& Plantio direto & 0,64 & 1,04 \\
& Integração & 0,59 & 1,14 \\
\hline \multirow{3}{*}{$0,05-0,10$} & Convencional & 0,59 & 1,16 \\
& Plantio direto & 0,57 & 1,14 \\
& Integração & 0,58 & 1,16 \\
\hline \multirow{3}{*}{$0,10-0,20$} & Convencional & 0,57 & 1,21 \\
& Plantio direto & 0,57 & 1,23 \\
& Integração & 0,59 & 1,11 \\
\hline
\end{tabular}

Para a camada de 0-0,05 m, o IHO foi igual à capacidade de água disponível no sistema convencional (Figura 1a). No sistema plantio direto (Figura 1b), a resistência à penetração limitou o intervalo hídrico ótimo para todos os valores de densidade. No sistema de integração, o IHO também teve a resistência à penetração como limite inferior para todos os valores de densidade, sendo essa limitação ainda maior que no sistema de plantio direto (Figura 1c), corroborando CONTE (2007).

A resistência à penetração na camada de $0 \mathrm{a} 5 \mathrm{~cm}$, menor no sistema convencional em relação aos sistemas com ausência de revolvimento (direto e integração), corrobora os resultados obtidos por BEUTLER et al. ( 2001), que compararam o sistema convencional, utilizando arado de discos com o sistema plantio direto. Os autores observaram, ainda, que a partir dessa profundidade as diferenças entre esses sistemas são pouco expressivas. 

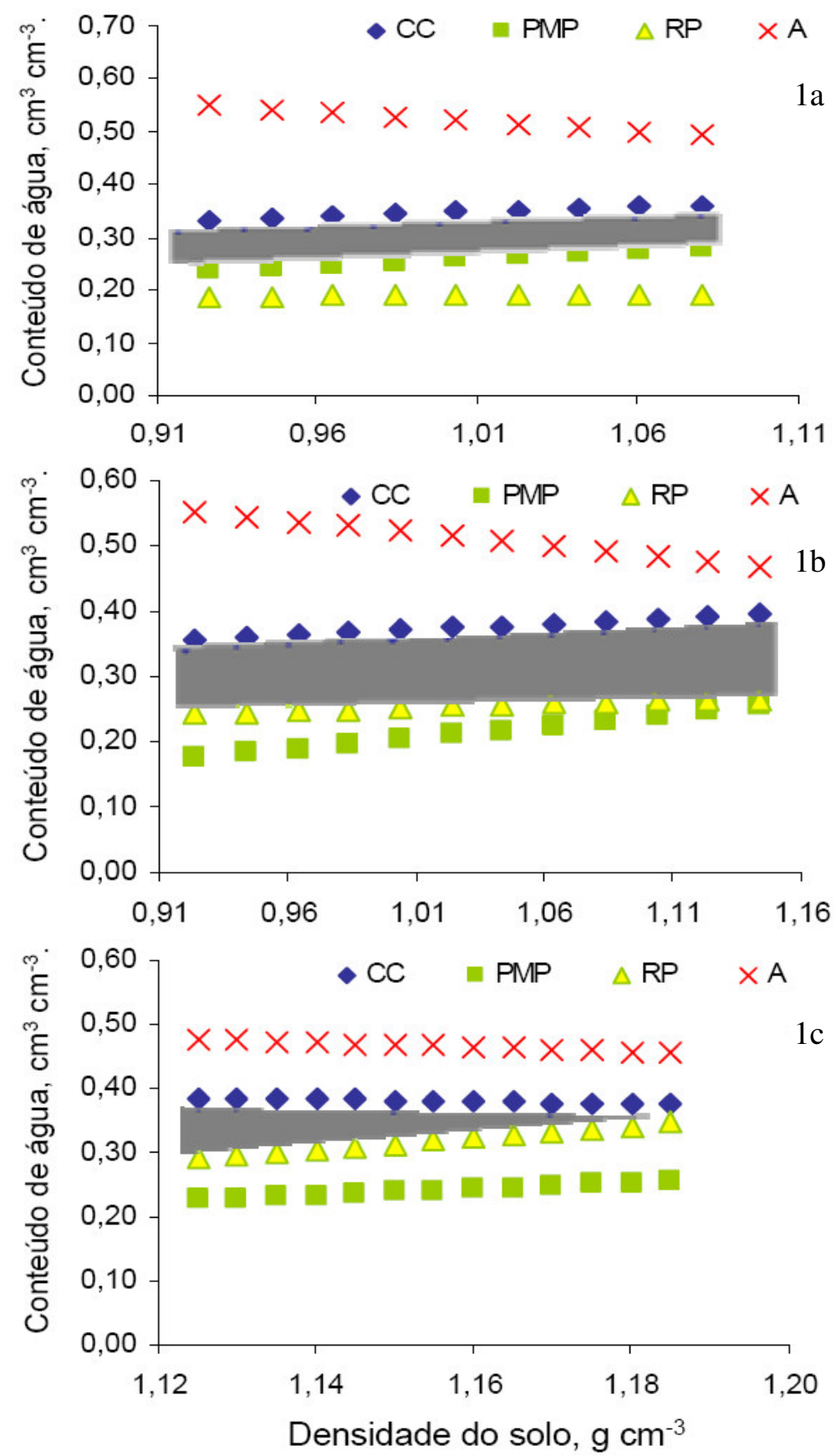

FIGURA 1. Variação da umidade volumétrica $\left(\mathrm{cm}^{-3} \mathrm{~cm}^{-3}\right)$ com a densidade do solo (Ds), nos limites críticos da capacidade de campo (CC), ponto de murcha permanente (PMP), porosidade de aeração de $10 \%$ (A) e resistência do solo à penetração de $2 \mathrm{MPa}(\mathrm{RP})$; na camada de 0 a $5 \mathrm{~cm}$ do sistema de cultivo convencional (a), direto (b) e integração (c). A área $r$ representa o Intervalo Hídrico Ótimo (IHO). Variation in water content $\left(\mathrm{cm}^{3} \mathrm{~cm}^{-3}\right)$ With the soil bulk density (Ds), in critical limits of field capacity (CC), Permanent wilting point, (MPP), porosity, aeration $10 \%$ (A) and resistance of the soil to the penetration of $2 \mathrm{MPa}(\mathrm{RP})$; in the layer of 0 to $5 \mathrm{~cm}$ of conventional system cultivation (a), direct (b) and integration (c). The rachurada area represents the Least Limiting Water Range (LLWR). 
Os três sistemas de cultivo tiveram IHO menor que a capacidade de água disponível na camada de 5-10 cm (Figuras 2a, 2b, 2c), devido à resistência à penetração. Nos sistemas plantio direto e convencional, a limitação foi mais acentuada (Figura 2b). No sistema convencional, o IHO teve como limite superior a porosidade de aeração quando a densidade era superior a $1,28 \mathrm{Mg} \mathrm{m}^{-3}$, indicando compactação severa, pois Latossolos oxídicos tendem a apresentar elevada porosidade sob condições naturais (FERREIRA et al., 1999).

Na camada de 0,10-0,20 m, a resistência à penetração foi o limite inferior do IHO para todos os valores de densidade, nos três sistemas de cultivo, indicando limitação severa à expansão radicular (Figuras 3a, 3b, 3c). A porosidade de aeração foi limitante sob valores de densidade mais elevados nos sistemas convencional e plantio direto por apresentar menos de $10 \%$ da porosidade total (Figura 3a e 3b).

A restrição física devido à resistência à penetração observada nas Figuras não condiz com relatos da literatura de melhoria de indicadores da qualidade do solo nas áreas com cultivo direto ou integração (FRANCHINI et al., 2000), as quais deveriam apresentar valores para seus atributos desejáveis com maior semelhança àqueles de ambientes naturais (DA ROS et al., 1997).

A adoção de valor de $2 \mathrm{MPa}$ como restritivo ao desenvolvimento radicular em áreas sob sistemas de cultivo conservacionistas parece ser um valor inadequado. Segundo TISDALL \& OADES (1982) e PEDROTTI et al., (2001), a resistência real exercida pelo solo à penetração radicular é, geralmente, menor que a resistência medida pelo penetrômetro, pois as raízes procuram os caminhos de menor resistência durante o seu crescimento.

A ausência de revolvimento dos sistemas de cultivo direto e integração proporciona a preservação e, consequentemente, aumento de poros gerados por raízes de plantas e por integrantes da mesofauna do solo. Segundo DEXTER (1998), para o desenvolvimento do sistema radicular, as plantas precisam explorar a porosidade presente no solo ou forçar a abertura de novos poros, superando a resistência oferecida pela matriz do solo. Esses poros preexistentes devem servir como caminhos preferenciais às raízes em expansão.

A arquitetura dos poros com maior continuidade e alinhamento nos sistemas plantio direto e integração, apresentando diâmetro acima de $10 \mu \mathrm{m}$, é favorável à expansão radicular, porém ignorada por hastes metálicas utilizadas nos testes de resistência à penetração, inúmeras vezes maior ao de uma raiz em expansão, estimando valores de resistência à penetração superiores aos realmente impostos pelo solo às raízes (GRANT \& LANFOND, 1993). Neste trabalho, a haste empregada foi de $4 \mathrm{~mm}$ de diâmetro, ou seja, $400 \mu \mathrm{m}$. 

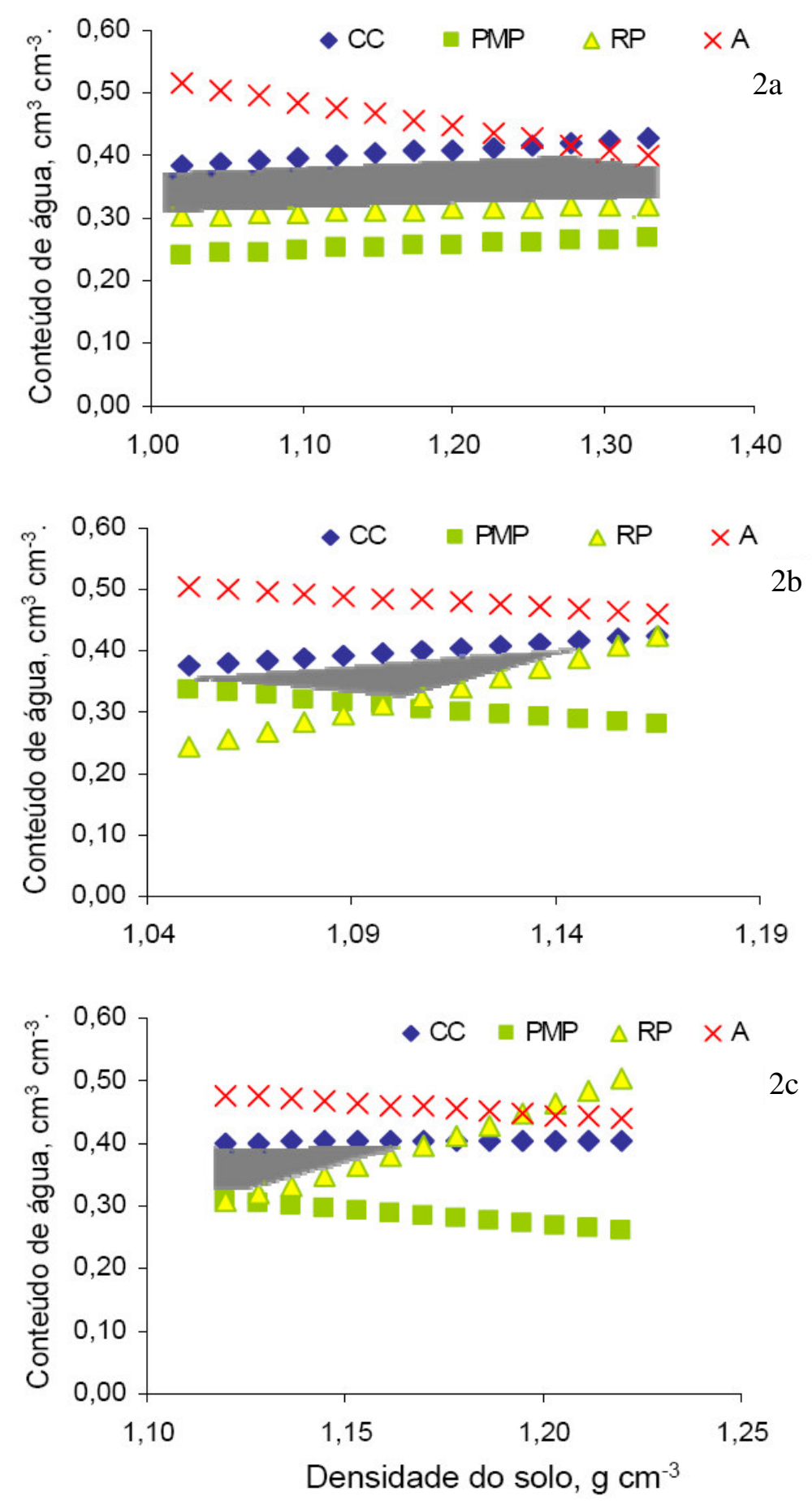

FIGURA 2. Variação da umidade volumétrica $\left(\mathrm{cm}^{3} \mathrm{~cm}^{-3}\right)$ com a densidade do solo (Ds), nos limites críticos da capacidade de campo (CC), ponto de murcha permanente (PMP), porosidade de aeração de $10 \%$ (A) e resistência do solo à penetração de $2 \mathrm{MPa}(\mathrm{RP})$; na camada de 5 a $10 \mathrm{~cm}$ do sistema de cultivo convencional (a), direto (b) e integração (c). A área rachurada representa o Intervalo Hídrico Ótimo (IHO). Variation in water content $\left(\mathrm{cm}^{3} \mathrm{~cm}^{-3}\right)$ with the soil bulk density (Ds), In critical limits of field capacity (CC), Permanent wilting point, (MPP), porosity, aeration $10 \%$ (A) and resistance of the soil to the penetration of $2 \mathrm{MPa}(\mathrm{RP})$; in the layer of 5 to $10 \mathrm{~cm}$ of conventional system cultivation (a), direct (b) and integration (c). The rachurada area represents the Least Limiting Water Range (LLWR). 

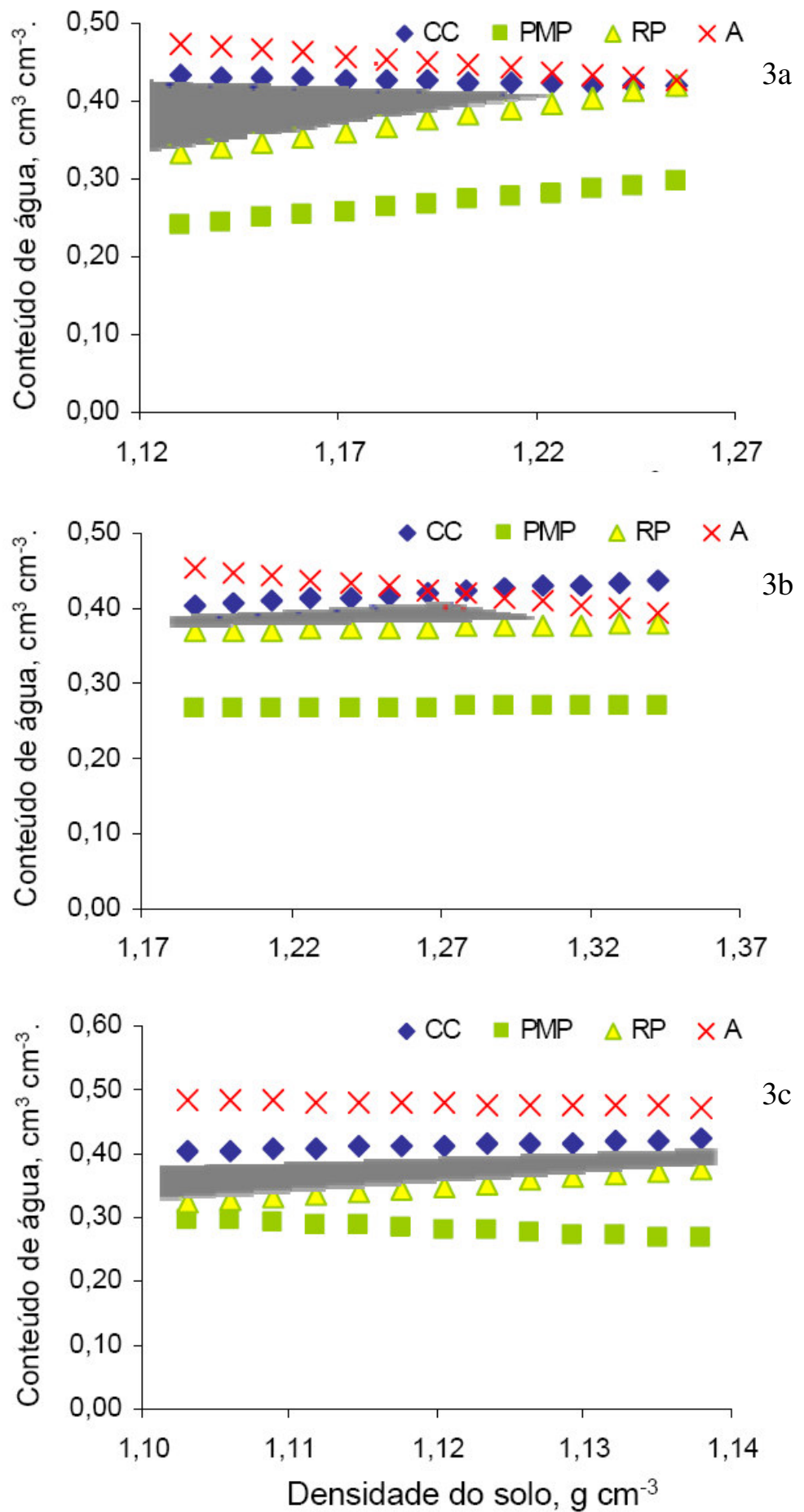

FIGURA 3. Variação da umidade volumétrica $\left(\mathrm{cm}^{3} \mathrm{~cm}^{-3}\right)$ com a densidade do solo (Ds), nos limites críticos da capacidade de campo (CC), ponto de murcha permanente (PMP), porosidade de aeração de $10 \%(\mathrm{~A})$ e resistência do solo à penetração de $2 \mathrm{MPa}(\mathrm{RP})$; na camada de 10 a $20 \mathrm{~cm}$ do sistema convencional (a), direto (b) e integração (c). A área rachurada representa o Intervalo Hídrico Ótimo (IHO). Variation in water content $\left(\mathrm{cm}^{3} \mathrm{~cm}^{-3}\right)$ with the soil bulk density (Ds), In critical limits of field capacity (CC), permanent wilting point, (MPP), porosity, aeration $10 \%$ (A) and resistance of the soil to the penetration of $2 \mathrm{MPa}(\mathrm{RP})$; in layer from 10 to $20 \mathrm{~cm}$ the conventional system (a), direct (b) and integration (c). The rachurada area represents the Least Limiting Water Range (LLWR). 


\section{CONCLUSÕES}

O conceito de IHO mostrou-se mais sensível que o conceito de água disponível, indicando restrição severa à expansão radicular quando considerado o valor de $2 \mathrm{MPa}$ como crítico. A resistência à penetração foi o limite inferior do IHO em oito das nove situações estudadas.

\section{AGRADECIMENTOS}

À Embrapa Agropecuária Oeste, Dourados - MS, por ter cedido a área experimental para a coleta das amostras. Ao CNPq, pelas bolsas de pesquisa concedidas.

\section{REFERÊNCIAS}

ARAÚJO, M.A.; TORMENA, C.A.; SILVA, A.P. Propriedades físicas de um Latossolo Vermelho distrófico cultivado e sob mata nativa. Revista Brasileira de Ciência do Solo, Viçosa, v.28, n.2, p.337-45, 2005.

BETZ, C.L.; ALLMARAS, R.R.; COPELAND, S.M.; RANDALL, G.W. Least limiting water range: traffic and long-term tillage influences in a webster soil. Soil Science Society of America Journal, Madison, v.62, n.62, p.1384-93, 1998.

BEUTLER, A.N.; SILVA, M.L.N.; CURI, N.; FERREIRA, M.M.; CRUZ, J.C.; PEREIRA FILHO, I.A. Resistência à penetração e permeabilidade de Latossolo Vermelho distrófico típico sob sistemas de manejo na região dos cerrados. Revista Brasileira de Ciência do Solo, Viçosa, v.25, p.167-77, 2001.

BUSSCHER, W.J. Adjustiment of flat-tipped penetrometer resistance data to a commom water content. Transactions of the ASAE, St. Joseph, v.33, n.3, p.519-24, 1990.

CAVALIERI, K.M.V.; TORMENA, C.A.; VIDIGAL FILHO, P.S.; GONÇALVES, A.C.A.; COSTA, A.C.S. Efeitos de sistemas de preparo nas propriedades físicas de um Latossolo Vermelho distrófico. Revista Brasileira de Ciência do Solo, Viçosa, v.30, n.1, p.137-47, 2006.

CONTE, O.; LEVIEN, R.; TREIN, C.R.; CEPIK, C.T.C.; DEBIASI, H. Demanda de tração em haste sulcadora na integração lavourapecuária com diferentes pressões de pastejo e sua relação com o estado de compactação do solo. Engenharia Agrícola, Jaboticabal, v.27, n.1, p.220-8, 2007.

DA ROS, C.O.; SECCO, D.; FIORIN, J.E.; PETRERE, C.; CADORE, M.A.; PASA, L. Manejo do solo a partir de campo nativo: efeito sobre a forma e estabilidade da estrutura ao final de cinco anos. Revista Brasileira de Ciência do Solo, Campinas, v.21, n.2, p.241-7, 1997.

DEXTER, A.R. Advances in characterization of soil structure. Soil Tillage Research, Amsterdam, v.11, p.199-238, 1998.

EMBRAPA. Centro Nacional de Pesquisa de Solos. Manual de métodos de análise de solo. 2.ed. rev. atual. Rio de Janeiro, 1997. 212 p. (Documentos, 1)

FERREIRA, M.M.; FERNANDES, B.; CURI, N. Influência da mineralogia da fração argila nas propriedades físicas de Latossolos da região Sudeste do Brasil. Revista Brasileira de Ciência do Solo, Lavras, v.23, p.515-24, 1999.

FIETZ, C.R.; URCHEI, M.A. Deficiência hídrica da cultura da soja na região de Dourados - MS. Revista Brasileira de Engenharia Agrícola e Ambiental, Campina Grande, v.6, n.2, p.262-5, 2002.

FRANCHINI, J.C.; BORKERT, C.M.; FERREIRA, M.M.; GAUDÊNCIO, G.A. Alterações na fertilidade do solo em sistemas de rotação de culturas em semeadura direta. Revista Brasileira de Ciência do Solo, Viçosa, v.24, n.2, p.459- 67, 2000.

GRABLE, A.R.; SIEMER, E.G. Effects of bulk density, agregate size, and soil water suction on oxigen diffusion, redox potencial and elongation of corns root. Soil Science Society America Proceedings, Madison, v.32, n.1, p.180-6, 1968. 
GRANT, C.A.; LANFOND, G.O. The effects of tillage systems and crop sequences on soil bulk density and penetration resistence on a clay soil in Southern Saskatchewan. Canadian Journal of Soil Science, Ottawa, v.73, n.2, p.223-32, 1993.

IMHOFF, S.; SILVA, P.A.; DIAS JUNIOR, M.S.; TORMENA, C.A. Quantificação de pressões críticas para o crescimento das plantas. Revista Brasileira de Ciência do Solo, Viçosa, v.25, p11-18, 2001.

KLUTE, A. Water retention: labotatory methods. In: KLUTE, A. (Ed.) Methods of soil analyses physical and mineralogical methods. $2^{\text {nd }}$ ed. Madison: ASA-SSSA, 1986. p.635-60.

LEÃO, T.P. Intervalo hídrico ótimo em diferentes sistemas de pastejo e manejo da pastagem. 2002. 58 f. Disssertação (Mestrado em Física do Solo) - Escola Superior de Agricultura "Luiz de Queiroz”, Universidade de São Paulo, Piracicaba, 2002.

LEITE, H.G.; OLIVEIRA, F.H.T. Statistical procedure to test identity between analytical methods. Communication in Soil Science Plant Analitical, Philadelphia, v.33, n.7/8, p.1.105-18, 2002.

LETEY, J. Relationship between soil physical properties and crop production. Advances Soil Science, New York, v.1, n.1, p.277-94, 1985.

PEDROTTI, A.; PAULETTO, E.A.; CRESTANA, S.; FERREIRA, M.M.; DIAS JÚNIOR, M.S.; GOMES, A.S.; TURATTI, A.L. Resistência mecânica do solo à penetração de um Planossolo submetido a diferentes sistemas de cultivo. Revista Brasileira de Ciência do Solo, Viçosa, v.25, n.3, p.521-9, 2001.

SILVA, A.P.; KAY, B.D. Estimating the least limiting water range of soil from properties and management. Soil Science Society of America Journal, Madison, v.61, p.877-83, 1997.

SILVA, A.P.; KAY, B.D.; PERFECT, E. Characterization of the least limiting water range. Soil Science Society of America Journal, Madison, v.58, p.1.775-81, 1994.

TAYLOR, H.M.; ROBERSON, G.M.; PARKER Jr., J.J. Soil strength-root penetration ralations to medium to coarse-textured soil materials. Soil Science, Madison, v.102, p.18-22, 1966.

TISDALL, J.M.; OADES, J.M. Organic matter and waterstable agregates in soils. Journal Soil Science, Oxford, v.33, n.1, p.141-63, 1982.

TORMENA, C.A.; ARAUJO, M.A.; FIDALSKI, J.; COSTA, M.J.Variação temporal do intervalo hídrico ótimo de um Latossolo Vermelho distroférrico sob sistemas de plantio direto. Revista Brasileira de Ciência do Solo, Viçosa, v.31, p.211-19, 2007.

TORMENA, C.A.; SILVA, A.P.; LIBARD, P.L. Caracterização do intervalo hídrico ótimo de um Latossolo Roxo sob plantio direto. Revista Brasileira de Ciência do Solo, Viçosa, v.22, p.573-81, 1998. 